Arthroskopie $2005 \cdot 18: 112-118$ DOI 10.1007/s00142-005-0297-z

Online publiziert: 8. März 2005

๑) Springer Medizin Verlag 2005

V. Valderrabano ${ }^{1,2} \cdot$ B. Hintermann ${ }^{2}$

${ }^{1}$ Human Performance Laboratory/Orthopaedics, University of Calgary, Kanada

${ }^{2}$ Orthopädisch-traumatologische Abteilung, Orthopädische Universitätsklinik Basel, Schweiz

\title{
Diagnostik und Therapie der medialen Sprunggelenkinstabilität
}

ner Rotationsinstabilität die mediale mit einer lateralen Instabilität kombiniert.

Als Entität der Sprunggelenkinstabilität ist die mediale Instabilität bislang wenig etabliert. Das Ziel der vorliegenden Arbeit ist es deshalb, eine Rationale für diesen Verletzungstyp zu erarbeiten und neben den klinischen Befunden eine Klassifikation und ein Therapiekonzept sowie deren Frühergebnisse darzustellen.

\section{Methode}

\section{Diagnostik}

einer schweren Distorsion entwickeln o$40 \%$ der Patienten, unabhängig von Qualität und Umfang konservativer Maßnahmen bei einer OSG-Distorsion, eine chronische Sprunggelenkinstabilität $[5,15]$. In einer arthroskopischen Untersuchung fanden Schäfer u. Hintermann [18] bei $21 \%$ der Patienten eine Beteiligung der medialen Bänder. In einer erweiterten prospektiven Untersuchung von 153 Patienten mit chronischer Sprunggelenkinstabilität fanden Hintermann et al. [14] später eine Inzidenz von $40 \%$ für die Begleitverletzung am medialen Bandapparat. Die Anamnese der 54 Patienten zeigte typischerweise ein zurückliegendes Pronations- und/oder Außenrotationstrauma mit Einwärtsdrehen des belasteten Beins sowie rezidivierendes "giving way"v. a. bei Ermüdung. Pathognomonische klinische Zeichen waren anteromediale Schmerzen sowie eine im Seitenvergleich erhöhte Pronationsstellung mit Valgusstellung des Rück-, aber fehlender Abduktionsfehlstellung des Vorfußes [14] In $77 \%$ der Fälle war mit dem Ausdruck ei-
Die Diagnose einer medialen Sprunggelenkinstabilität wird auf der Basis von Anamnese, klinischer Untersuchung mit spezifischen Tests, konventionellem Röntgen und Arthroskopie gestellt (• Tabelle 1).

Anamnestisch ist ein Status nach einem gnomonisch. Häufig beschreiben die $\mathrm{Pa}$ tienten auch multiple OSG-Distorsionen, ohne den spezifischen Verletzungsmechanismus eruieren zu können, oder gar ein Supinationstrauma. Typische Verletzungssituationen sind die Läsionen des medialen Bandkomplexes während einer sportlichen Betätigung, beim Bergabgehen, beim Treppensteigen oder beim Landen auf unebenem Terrain. Die Patienten mit einer chronischen medialen Instabilität beschreiben typischerweise ein mediales oder anteromediales "giving way" beim Bergabgehen oder Treppen hinunterstei-

\section{Anamnese} Pronations- oder Eversionstrauma patho- gen und anteromediale OSG-Schmerzen. Später bei der allmählichen Mitbeteiligung und Instabilität des lateralen Bandkomplexes (Rotationsinstabilität) sowie der Zunahme der Rückfußvalgusdeformität klagen die Patienten auch über anterolaterale OSG-Schmerzen.

\section{Klinische Untersuchung}

Bei voller Belastung durch das Körpergewicht des Patienten werden unter Beach-

\begin{tabular}{|c|c|}
\hline \multicolumn{2}{|c|}{$\begin{array}{l}\text { Diagnostik der medialen } \\
\text { Sprunggelenkinstabilität }\end{array}$} \\
\hline Anamnese $^{a}$ & $\begin{array}{l}\text { - Zustand nach Pronations- } \\
\text { (Eversions-)Trauma } \\
\text { •"Giving way“ } \\
\text { - Anteromedialer } \\
\text { Sprunggelenksschmerz }\end{array}$ \\
\hline $\begin{array}{l}\text { Klinische } \\
\text { Unter- } \\
\text { suchung }^{\text {a }}\end{array}$ & $\begin{array}{l}\text { - Schubladentest } \\
\text { (Talusvorschub) } \\
\text { - Valgusstresstest } \\
\text { - Quantifizierung des } \\
\text { Valgusmalalignements } \\
\text { - Einbeinzehenspitzenstand } \\
\text { (falls normale Rückfuß- } \\
\text { varisation: Ausschluss } \\
\text { begleitender Tibialis- } \\
\text { posterior-Dysfunktion) }\end{array}$ \\
\hline \multicolumn{2}{|c|}{ Konventionelles Röntgen (im Stehen) ${ }^{\mathrm{a}}$} \\
\hline \multicolumn{2}{|c|}{ Magnetresonanztomographie } \\
\hline \multicolumn{2}{|c|}{ Dynamische Pedobarographie } \\
\hline Diagnostisch & e Arthroskopie \\
\hline
\end{tabular}




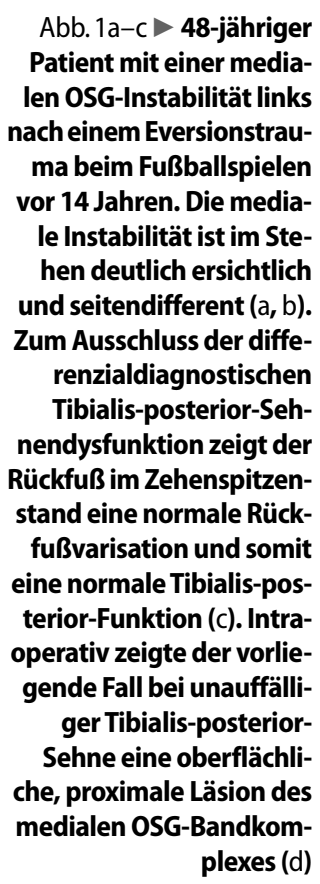

tung von potenziellem Valgusmalalignement, Pronationsdeformität und/oder Innenrotation des Beins, Asymmetrie der Malleolen und Schwellung des medialen und/oder lateralen Sprunggelenks, Form und Position beider Füße untersucht (• Abb. 1). Eine isolierte ligamentäre mediale Sprunggelenkinstabilität weist ein Valgusmalalignement auf, das im Einbeinzehenspitzenstand eine normale Varisation des Rückfußes aufweist. Eine ligamentäre mediale Sprunggelenkinstabilität mit kombinierter Tibialis-posterior-Dysfunktion weist ein Valgusmalalignement auf, das im Einbeinzehenspitzenstand im Rückfußvalgus verbleibt. Ein Valgusmalalignement des Rückfußes ist definiert als eine asymmetrische Pronationsdeformität des betroffenen Fußes mit exzessivem Valgus des Rückfußes, Pronation des Fußes und häufig Innenrotation des Beins. Kriterien für eine sekundär begleitende Dysfunktion des M. tibialis posterior sind das Vorhandensein einer Abflachung des medialen Längsgewölbes, ein zunehmender Rückfußvalgus und eine Vorfußabduktion, die nicht durch einen Einbeinzehenspitzenstand (,single heel rise test“) korrigiert werden können. Patienten mit einer Tarsalkoalitio weisen häufig eine Valgusrückfußdeformität mit variablem Aus$\mathrm{maß}$ an Subtalarbeweglichkeit, jedoch ei- ner generellen Abnahme der Rückfußflexibilität auf.

Druckdolenz im anteromedialen/medialen Sprunggelenk ist das palpatorische Hauptmerkmal der medialen Sprunggelenkinstabilität. Dieses Symptom scheint einerseits durch die verletzten Bänder selbst, andererseits aber auch durch die in der Arthroskopie häufig sichtbare mediale Synovialitis bedingt zu sein. Als palpatorische Zeichen einer möglichen begleitenden Tibialis-posterior-Dysfunktion gelten jedoch der perimalleoläre Druckschmerz im Bereich der Tibialis-posterior-Sehne im Anfangsstadium sowie der anterolaterale Sprunggelenkschmerz im fortgeschrittenen Stadium dieser progressiven Rückfußvalgusdeformität.

Einzehenspitzenstand. Zur Bestätigung der Tibialis-posterior-Dysfunktion kann neben dem Einzehenspitzenstand (Rückfuß verbleibt in Valgusposition) die klinische, grobe isometrische Inversionskraftmessung des M. tibialis posterior in leichter Plantarflexion herangezogen werden.

Vorderer Schubladentest. Der vordere Schubladentest (Talusvorschub) sollte beim sitzenden Patienten mit hängendem und in $90^{\circ}$ flektiertem Bein durchgeführt werden. Während eine Hand die distale
Tibia fixiert, greift die andere Hand den Rückfuß an Talushals und Ferse. Hierbei versucht der Untersucher, den Talus relativ zur Tibia nach vorne zu verschieben. Bei der medialen Sprunggelenkinstabilität zeigt sich eine seitendifferente, pathologische anteriore Translation des Talus, die zunimmt, wenn der Fuß relativ zur Tibia graduell innenrotiert wird. Im Falle einer mediolateralen Sprunggelenkinstabilität (Rotationsinstabilität) liegt eine ausgeprägtere und generelle anteriore Talustranslation vor.

\section{Röntgen, CT und MRT}

Konventionelles Röntgen im Stehen gehört zur Basisdiagnostik der medialen Sprunggelenkinstabilität (• Tabelle 1). Hierbei werden anteroposteriore und laterale Aufnahmen des Sprunggelenks und Fußes zum Ausschluss knöcherner Pathologien (Avulsionen, Frakturen) sowie zur Bestimmung des anteroposterioren und lateralen Talo-Metatarsale-1-Winkels veranlasst. Falls eine talokalkaneare oder die häufigere kalkaneonavikulare Koalitio sowie eine knöcherne Fragmentation oder Fraktur vermutet werden, ist eine Computertomographie gerechtfertigt. Die traditionell durchgeführten Stressröntgenaufnahmen werden generell von den Autoren nicht mehr empfohlen, da diese kei- 
Arthroskopie $2005 \cdot 18: 112-118$

DOI 10.1007/s00142-005-0297-z

(c) Springer Medizin Verlag 2005

V. Valderrabano $\cdot$ B. Hintermann

\section{Diagnostik und Therapie der medialen Sprunggelenkinstabilität}

\section{Zusammenfassung}

Die mediale Sprunggelenkinstabilität liegt typischerweise nach einem Pronationstrauma des Rückfußes vor. Dominante Symptome sind ein Unsicherheitsgefühl sowie ein mediales "giving way" beim Berg- und Treppabgehen, während in der klinischen Untersuchung ein anteromedialer OSGSchmerz und eine im Seitenvergleich erhöhte Pronationsstellung typisch sind. Für die Diagnosestellung dienen anamnestischen Angaben, klinische Untersuchung und konventionelles Röntgen unter Belastung. Während die MRT-Untersuchung eher selten benutzt wird, hat sich die Arthroskopie als sehr hilfreich für die Diagnosesicherung erwiesen. In Anlehnung an die derzeitigen Klassifikationen war die am häufigsten vorliegende mediale Sprunggelenkinstabilität das Stadium III in der klinischen Klassifikation, Stadium 3 in der arthroskopischen sowie Stadium A in der lokoanatomischen Klassifikation.

\section{Schlüsselwörter}

Sprunggelenk · Deltoid · Instabilität · Arthroskopie

\section{Diagnostics and therapy of medial ankle instability}

\begin{abstract}
Medial ankle instability typically occurs following pronation trauma involving the hindfoot. The predominant symptoms include a feeling of uncertainty and medial giving way while descending an incline or stairs whereas clinical examination characteristically reveals anteromedial ankle pain and increased pronation compared to the contralateral side. The diagnosis rests on the patient's history, clinical examination, and conventional stress $\mathrm{X}$-ray. While magnetic resonance imaging is rarely employed, arthros-
\end{abstract}

copy has proven to be very effective for determining the diagnosis. Based on classification systems currently in use, the most frequently observed medial ankle instability was stage III in the clinical classification, stage 3 in the arthroscopic classification, and stage $A$ in the locomotive anatomic classification.

\section{Keywords}

Ankle $\cdot$ Deltoid $\cdot$ Instability $\cdot$ Arthroscopy ne Zusatzinformationen liefern, sondern eine zusätzliche Schädigung verursachen können.

Ein weiteres und oft hilfreiches Diagnostikum ist die dynamische Pedobarographie, die mit einem dynamischen plantaren Druckbild das Abrollverhalten des Fußes objektiv darstellt. Die Magnetresonanztomographie (MRT) ist ein weiteres sekundäres und nichtinvasives Hilfsmittel zur Diagnostik der Läsion des medialen Sprunggelnkligamentkomplexes sowie zum Ausschluss einer begleitenden chondralen Läsion oder Tibialis-posterior-Sehnendegeneration.

\section{Arthroskopie}

Im Falle der medialen Sprunggelenkinstabilität stellt die OSG-Arthroskopie eine essenzielle Basisdiagnostik zur Diagnosesicherung und vollständigen präoperativen Evaluation dar (s. Abb. 4 im Beitrag Schneiderbauer et al. in dieser Ausgabe). Die OSG-Arthroskopie wird in der orthopädischen Universitätsklinik Basel durch einen zentralen Zugang und unter Verwendung eines $\mathrm{CO}_{2}$-Mediums sowie eines 40-mm-Arthroskops mit $30^{\circ}$-Optik durchgeführt. Da die meisten medialen Ligamentläsionen im proximalen Insertionsbereich lokalisiert sind, ist deren Pathologie am häufigsten durch das Vorliegen einer frei liegenden Insertionszone am medialen Malleolus oder am lateralen Malleolus im Falle einer Rotationsinstabilität ersichtlich (• Tabelle 2). Weitere typische und häufige Arthroskopiebefunde der medialen Sprunggelenkinstabilität sind anteromediale Synovialitiden, Periostnarben, Avulsionen, Bandelongationen und osteophytäre Ausziehungen (• Tabelle 2). Die Arthroskopie ermöglicht durch die intraartikuläre funktionelle Analyse das Instabilitätsmuster der verletzten Bänder zu eruieren, dies geschieht durch

- einen manuellen axialen Zug (tibiotalarer Abstand; • Tabelle 2) zur Quantifizierung der Gelenklaxität,

- den Talusvorschub zur Bestimmung der anteromedialen oder anterioren Instabilität,

- einen Valgusstress zur Prüfung des medialen Bandkomplexes und

- einen Varusstress zur Prüfung des lateralen Bandkomplexes. 
Tabelle 2

Klassiffkationen der medialen Sprunggelenkinstabilität*

Klinische Klassifikation I-IV

\begin{tabular}{|c|c|c|c|c|c|c|c|c|c|c|}
\hline & $\begin{array}{l}\text { Giving } \\
\text { way }\end{array}$ & \multicolumn{2}{|c|}{$\begin{array}{l}\text { Rückfußvalgus/ } \\
\text { Pronation }\end{array}$} & \multicolumn{2}{|c|}{$\begin{array}{l}\text { Medialer } \\
\text { OSG-Schmerz }\end{array}$} & \multicolumn{2}{|c|}{$\begin{array}{l}\text { Anterolateraler } \\
\text { OSG-Schmerz }\end{array}$} & \multicolumn{2}{|c|}{$\begin{array}{l}\text { Tibialis-posterior- } \\
\text { Dysfunktion }\end{array}$} & $\begin{array}{l}\text { Deformitäts- } \\
\text { korrekturfähigkeit }\end{array}$ \\
\hline Stadium I & + & \multicolumn{2}{|l|}{+} & \multicolumn{2}{|l|}{+} & \multicolumn{2}{|l|}{$(+)$} & \multicolumn{2}{|l|}{-} & Ja \\
\hline Stadium II & ++ & \multicolumn{2}{|l|}{+} & \multicolumn{2}{|l|}{+++} & \multicolumn{2}{|l|}{$(+)$} & \multicolumn{2}{|l|}{-} & Ja \\
\hline Stadium III & +++ & \multicolumn{2}{|l|}{++} & \multicolumn{2}{|l|}{+++} & \multicolumn{2}{|l|}{+} & \multicolumn{2}{|l|}{+} & Nein \\
\hline Stadium IV & ++++ & \multicolumn{2}{|l|}{+++} & \multicolumn{2}{|l|}{++++} & \multicolumn{2}{|l|}{++} & \multicolumn{2}{|l|}{++} & Nein \\
\hline \multicolumn{11}{|c|}{ Arthroskopische Klassifikation 1-4 } \\
\hline & \multicolumn{2}{|c|}{$\begin{array}{l}\text { Oberflächliche/ } \\
\text { ventrale } \\
\text { Deltoidanteile }\end{array}$} & \multicolumn{2}{|c|}{$\begin{array}{l}\text { Tiefe/posteriore } \\
\text { Deltoidanteile }\end{array}$} & \multicolumn{2}{|c|}{$\begin{array}{l}\text { Mediale } \\
\text { malleoläre } \\
\text { Periostnarbe }\end{array}$} & \multicolumn{2}{|c|}{$\begin{array}{l}\text { Mediale malleoläre } \\
\text { Osteophyten }\end{array}$} & $\begin{array}{l}\text { Tibiotalarer } \\
\text { Abstand } \\
(\mathrm{mm})\end{array}$ & $\begin{array}{l}\text { Laterale OSG- } \\
\text { Bandläsion }\end{array}$ \\
\hline Stadium 1 & \multicolumn{2}{|c|}{$\begin{array}{l}\text { Elongiert/partiell ge- } \\
\text { rissen/Avulsion }\end{array}$} & \multicolumn{2}{|c|}{ Normal } & \multicolumn{2}{|l|}{+} & \multicolumn{2}{|l|}{+} & $2-5$ & Nein \\
\hline Stadium 2 & \multicolumn{2}{|c|}{ Gerissen } & \multicolumn{2}{|c|}{$\begin{array}{l}\text { Elongiert/partiell } \\
\text { gerissen }\end{array}$} & \multicolumn{2}{|l|}{++} & \multicolumn{2}{|l|}{++} & $2-5$ & Nein \\
\hline Stadium 3 & \multicolumn{2}{|c|}{ Gerissen } & \multicolumn{2}{|c|}{$\begin{array}{l}\text { Elongiert/partiell } \\
\text { gerissen }\end{array}$} & \multicolumn{2}{|l|}{+++} & +++ & & $>5$ & Ja \\
\hline Stadium 4 & Geri & & Geris & & +++ & & ++++ & & $>5$ & Ja \\
\hline Chirurgisch & lokoana & ische Klassifil & kation & & & & & & & \\
\hline Stadium A & $\begin{array}{l}\text { Prox } \\
\text { Ligg }\end{array}$ & $\begin{array}{l}\text { ler Riss des } \mathrm{m} \\
\text { iotalare anter }\end{array}$ & $\begin{array}{l}\text { ediale } \\
\text { ior un }\end{array}$ & $\begin{array}{l}\text { GG-Bandkc } \\
\text { sterior, Lic }\end{array}$ & $\begin{array}{l}\text { plexe } \\
\text { ibioca }\end{array}$ & $\begin{array}{l}\text { oroxima } \\
\text { aneale }\end{array}$ & Inteile c & ig. tibiona & culare und Tibi & pringligament, \\
\hline Stadium B & $\begin{array}{l}\text { Inte } \\
\text { Liga }\end{array}$ & $\begin{array}{l}\text { diärer Riss de } \\
\text { tts }\end{array}$ & 5 medi & OSG-Ban & mple & s: interr & iäre An & des Lig. ti & onaviculare un & Tibiospring- \\
\hline Stadium C & $\begin{array}{l}\text { Dist } \\
\text { Spri }\end{array}$ & $\begin{array}{l}\text { iss des medi } \\
\text { ament }\end{array}$ & alen 0 & andkom & es: $d$ & ale Ante & Lig. & navicular & nd Tibiospring & aments, \\
\hline
\end{tabular}

*Nach Hintermann et al. [9, 12] und Schäfer u. Hintermann [18]

Zuletzt ermöglicht die OSG-Arthroskopie noch die direkte Erkennung und Therapie der häufig vorhandenen Knorpelbegleitläsionen durch

- Entfernung freier Gelenkkörper,

- arthroskopisches Debridement, Mikrofrakturing oder andere Techniken.

In manchen Fällen vermag die OSG-Arthroskopie die bei der medialen Sprunggelenkinstabilität häufig sichtbaren MRTKnorpel-Signalalterationen als nichtpathologische Befunde zu differenzieren und dadurch auch eine unnötige Knorpelbehandlung zu vermeiden. Generell liegt arthroskopisch eine mediale Sprunggelenkinstabilität vor, wenn die medialen Bandstrukturen isoliert eine Pathologie aufweisen. Eine Rotationsinstabilität liegt vor, wenn sowohl die medialen als auch die lateralen Bandstrukturen kombiniert eine Pathologie aufweisen.

\section{Klassifikationen}

Hintermann et al. $[13,14]$ und Schäfer u. Hintermann [18] haben in früheren Arbeiten die mediale Sprunggelenkinstabilität auf klinischem, arthroskopischem und lokoanatomischem Niveau klassifiziert (• Tabelle 2). Die klinische Klassifikation basiert auf der Erkennung und dem Ausmaß des „giving way“, der Rückfußvalgus-/Pronationsdeformität, des medialen und lateralen OSG-Schmerzes, der Tibialis-posterior-Sehneninsuffizienz und der Deformitätskorrekturfähigkeit. Die arthroskopische Klassifikation beurteilt die medialen Bänder nach dem ligamentären Läsionsmuster, dem Vorhandensein von Periostnarben und Osteophyten, dem tibiotalaren Abstand sowie der Beteilung des lateralen Bandkomplexes. Die chirurgische lokoanatomische Klassifikation zum Zeitpunkt der Rekonstruktion definiert die Lage des vorliegenden Risses als proximal, intermediär oder distal.

\section{Differenzialdiagnose}

Zu den Differenzialdiagnosen der medialen Sprunggelenkinstabilität gehören:

- Tibialis-posterior-Sehnendysfunktion/ Knick-Senk-Fuß,

- Coalitio talocalcanearis oder talonaviculare,

- posttraumatische und neurologische Erkrankungen, die eine Rückfußvalgusdeformität verursachen.

\section{Operatives Behandlungskonzept}

\section{Rekonstruktion des medialen Bandkomplexes}

Die Rekonstruktion des medialen Bandkomplexes ist dann indiziert, wenn eine persistierende, symptomatische und klinisch verifizierte mediale Sprunggelenkinstabilität vorliegt. 
Die frühe Rekonstruktion der verletzten Bänder hat den großen Vorteil, dass eine normale Anatomie vorliegt und assoziierte Komorbiditäten wie z. B. chondrale Läsionen oder gar arthritische Veränderungen fehlen. Die Bänder können abhängig von der vorliegenden Gewebequalität direkt oder durch Grafts rekonstruiert werden.

Durch einen medialen OSG-Zugang mit einer Inzision vom medialen Malleolus zum Os naviculare ziehend werden die mediale Bänderanteile, die Tibialis-posterior-Sehnen, das Springligament sowie der mediale Malleolus dargestellt. In situ wird zwischen einem proximalen (A), intermediären (B) oder distalen Riss (C) des medialen Bandkomplexes differenziert ( $\bullet$ Tabelle 2). Bei genügender Bandqualität, wie im Falle einer früherkannten medialen Sprunggelenkinstabilität, wird eine Direktrekonstruktion angestrebt. Hierbei wird der ventrale Anteil des medialen Malleolus angefrischt und 2 Knochenanker (z. B. Mitek) platziert, die zur Naht, Rekonstruktion, Raffung und somit der Kontinuitätswiederherstellung der lädierten Bänder dienen. Resorbierbare Fäden der Größe o werden zum sorgfältigen Verschluss des Bandkomplexes benutzt. Bei einer insuffizienten Gewebequalität, wie z. B. im Falle einer persistierenden und fortgeschrittenen medialen Sprunggelenkinstabilität, sind eine direkt Rekonstruktion mit Mitek-Ankern häufig nicht möglich und eine autologe Sehnengraftrekonstruktion nötig. Die Autoren bevorzugen hier die Verwendung des freien Plantarissehnengrafts, der in anatomischer Durchflechtungsart die insuffizienten Bänder ersetzend eingeführt und fixiert wird. Hierbei werden 2 konvergierende 3,2-mmBohrlöcher am ventralen medialen Malleolus gesetzt, 2-8 $\mathrm{mm}$ proximal der Malleolenspitze. Analog wird ein dorsoplantarer Tunnel am Os naviculare gebohrt. Der Plantarissehnengraft wird dann von kranial nach kaudal durch den malleolären und navikulären Tunnel gezogen. Während der Fuß in neutraler Position gehalten wird, werden die Enden des Grafts unter leichter Spannung mit einem resorbierbaren Faden der Größe o aneinander genäht. Abschließend werden die noch verbliebenen ursprünglichen Ligamentreste zum Transplantat hin vernäht, womit eine starke Rekonstruktion des Lig. tibionaviculare sowie des Tibiospringligaments kreiert wird.

\section{Rekonstruktion des lateralen OSG-Bandkomplexes}

Bei einer sog. Rotationsinstabilität, d. h. kombinierten medialen und lateralen Instabilität, ist die Rekonstruktion des lateralen OSGBandkomplexes [9] in der gleichen Sitzung indiziert. Diese Situation ist nach der Erfahrung der Autoren sehr häufig und kann sowohl klinisch als auch mittels diagnostischer Arthroskopie erkannt werden. Die Erkrankung kann dadurch erklärt werden, dass unter Belastung die mediale Sprunggelenkinstabilität eine repetitive laterale Translation des Talus verursacht und im Verlauf zu einer sekundären Insuffizienz des lateralen OSG-Bandkomplexes führt. Durch die hohe Inzidenz der Knorpelläsionen am medialen Talus sowie des uniformen Verletzungsmusters der lateralen Bänder wird diese Hypothese bekräftigt: die Ligg. fibulotalare anterius sowie fibulocalcaneare sind von der gemeinsamen Insertion im distalen Anteil des lateralen Malleolus zusammen abgelöst.

\section{Tibialis-posterior- \\ Sehnenrekonstruktion}

Eine Tibialis-posterior-Sehnenrekonstruktion (Raffung, primäre Sehnennaht, Refixation am Os naviculare, FDL-Transfer; [8, 2o]) ist im Falle einer sichtbaren Degeneration, Elongation, Teil- oder Komplettruptur und Insertionsavulsion indiziert.

\section{Laterale Kalkaneusverlängerungs- osteotomie}

Eine laterale Kalkaneusverlängerungsosteotomie [12] ist bei einer ausgeprägten Rückfußvalgus- oder Pronationsdeformität sowie einer schweren Läsion des medialen OSGBandkomplexes angezeigt. Eine solche Osteotomie ist auch dann indiziert, wenn eine vorbestehende Valgus- oder Pronationsdeformität (wenn z. B. eine Valgus- oder Pronationsdeformität an der kontralateralen, asymptomatischen Seite vorhanden ist) und/oder ein chronisches Tarsaltunnelsyndrom vorliegt. Durch die Korrektur der ossären Deformität vermag diese Osteotomie die rekonstruierten medialen Bänder indirekt von pathologischem Stress zu entlasten und die Biomechanik und Funktion der Muskelsehneneinheiten zu verbessern.

\section{Talonavikulare Arthrodese}

Eine talonavikulare (TN-)Arthrodese ist bei einer exzessiven medialen Sprungge- lenkinstabilität indiziert, d. h. bei einem signifikanten Defekt des tibionavikularensowie Springligaments. Während eine ligamentäre und ossäre Rekonstruktion bei sportlich aktiven Patienten und Athleten zu bevorzugen ist, stellt eine TN-Arthrodese bei adipösen, nicht aktiven Patienten, die einen schmerzlosen und stabilen Fuß wünschen, die Therapie der Wahl dar.

\section{Postoperatives Management}

Zur postoperativen Behandlung der medialen Sprunggelenkinstabilität gehören eine Schienenbehandlung sowie eine konsequente Nichtbelastung, bis die Schwellung abgeklungen ist, normalerweise eine Woche. Danach wird im Falle einer reinen Weichteilrekonstruktion ein Stabilschuh (z. B. Künzli-Stabilschuh, Künzli-Schuhfabrik, Windisch, Schweiz) oder im Falle einer zusätzlichen Osteotomie oder Arthrodese ein Gehgips mit einem verstärkten medialen Gewölbesupport in Neutralposition angepasst. Diese werden während 5 Wochen getragen, im Falle einer TN-Arthrodese sogar 7 Wochen. Nach Gipsentfernung wird ein intensives Rehabilitationsprogramm verordnet:

\section{- Verbesserung der Beweglichkeit, \\ - Stärkung der Tibialis-posterior- sowie der Peronealmuskulatur und der neu- romuskulären Kontrolle.}

Die physiotherapeutische Betreuung sollte bis zum Erreichen einer objektiv akzeptablen Kraft und Propiozeption fortgesetzt werden.

\section{Kasuistik}

Seit 1994 wurden an der orthopädischen Universitätsklinik Basel insgesamt 382 konsekutive Patienten mit einer chronischen Instabilität des OSG vor der geplanten Bandrekonstruktion operativ versorgt. $48 \mathrm{~Pa}$ tienten wurden wegen einer posttraumatischen ossären Deformität und/oder Arthrose (Gelenkinkongruenz unter Belastung, Osteophyten) und 70 Patienten wegen einer Voroperation an den Bändern ausgeschlossen. Das untersuchte Kollektiv umfasste 264 Patienten, wovon 17 beidseitig behandelt wurden. Die insgesamt 281 Sprunggelenke betrafen 188 Frauen und 
Tabelle 3

Typverteilung der medialen

Sprunggelenkinstabilität ${ }^{\mathrm{a}}$

\begin{tabular}{|lrr}
\multicolumn{2}{c}{$\mathbf{n}$} & $\%$ \\
Klinische Stadien I-IV & & \\
Stadium I & 9 & 6 \\
Stadium II & 21 & 15 \\
Stadium III & 106 & 76 \\
Stadium IV & 4 & 3 \\
\hline
\end{tabular}

Arthroskopische Stadien 1-4

$\begin{array}{lll}\text { Stadium } 1 & 16 & 11\end{array}$

$\begin{array}{lll}\text { Stadium } 2 & 21 & 15\end{array}$

$\begin{array}{lll}\text { Stadium } 3 & 86 & 62\end{array}$

$\begin{array}{lll}\text { Stadium } 4 & 17 & 12\end{array}$

Lokoanatomische Stadien A-C

$\begin{array}{llr}\text { Stadium A } & 77 & 55 \\ \text { Stadium B } & 51 & 36 \\ \text { Stadium C } & 12 & 9\end{array}$

a Vergleiche - Tabelle 2.

93 Männer mit einem Durchschnittsalter von 35,8 (15-61) Jahren. In sämtlichen Fällen lag eine symptomatische Instabilität des OSG nach einer mindestens 3-monatigen erfolglosen konservativen Behandlung vor. Der Abstand zur letzten Untersuchung betrug mindestens ein Jahr.

\section{Ergebnisse}

Im Patientenkollektiv der chronischen Sprunggelenkinstabilität wurden 140 Fälle $(50 \%)$ mit einer Läsion des medialen Bandkomplexes beobachtet. Davon wiesen 103 (74\%) eine mediale Bandläsion, kombiniert mit einer Verletzung der lateralen Bänder (Rotationsinstabilität), auf. In 37 Fällen (26\%) lag eine isolierte mediale Bandkomplexläsion vor. Die durchschnittliche Nachbeobachtungszeit betrug 3,6 (19 Jahre). Die subjektive Zufriedenheit wurde von den Patienten wie folgt angegeben:

- sehr zufrieden $42 \%$,

- zufrieden $48 \%$,

- moderat $7 \%$,

- unzufrieden $3 \%$.

Der funktionelle Zustand, erhoben durch den AOFAS-Hindfoot-Score (o-10o Punkte; [16]), konnte von 41 (17-50 Punkten) präoperativ auf 91 (78-10o Punkte) beim aktuellen follow-up verbessert werden. Die am häufigsten vorliegende mediale Sprungge- lenkinstabilität war das Stadium III in der klinischen, Stadium 3 in der arthroskopischen sowie Stadium A in der lokoanatomischen Klassifikation (• Tabelle 3; • Abb. 1).

\section{Diskussion}

Über die Entität der medialen Sprunggelenkinstabilität ist wenig bekannt $[2,7,14]$. Der pathomechanische Zusammenhang zwischen Verletzungen des medialen und lateralen Bandkomplexes ist teilweise noch nicht geklärt, auch nicht die primäre oder sekundäre Koexistenz einer Tibialis-posterior-Dysfunktion im Rahmen der medialen Sprunggelenkinstabilität [13, 14]. Es bestehen Hinweise, dass der Knick-Senk-Fuß häufiger eine mediale Bandverletzung erleidet, also möglicherweise ein begünstigender Faktor bei der Entstehung einer medialen Bandverletzung sein könnte $[1,6,11]$. Spezifische Studien mit Einschluss biomechanischer Analysen sind notwendig, um diese Zusammenhänge zu klären.

\section{Akute mediale Bandverletzung}

Dagegen existiert ein konkret nachgewiesener Zusammenhang zwischen der akuten medialen Bandverletzung und der Entwicklung einer späteren medialen Sprunggelenkinstabilität [14]. Das anamnestische Hauptmerkmal ist hierbei ein Status nach einem Pronations- oder Eversionstrauma. Häufig beschreiben die Patienten auch multiple OSG-Distorsionen, ohne den spezifischen Verletzungsmechanismus eruieren zu können. Die vorliegende Arbeit unterstreicht besonders die Tatsache, dass eine mediale sehr häufig mit einer lateralen Sprunggelenkinstabilität vergesellschaftet sein (74\%), d. h. eine Rotationsinstabilität vorliegen kann. Die Frage, ob die mediale Instabilität hierbei die laterale begünstigt oder umgekehrt, kann mit dieser Studie nicht beantwortet werden. Hintermann et al. [14] beschrieben, dass $42 \%$ der Patienten mit medialer Instabilität anamnestisch ein Supinationstrauma bestätigten und im Verlauf doch eine mediale Instabilität entwickelten.

\section{Diagnosestellung}

Anamnese, sorgfältige klinische Untersuchung, konventionelles Röntgen unter Belastung sowie Arthroskopie sind die wichtigsten Mittel bei der Diagnosestellung der medialen Sprunggelenkinstabilität und gelten als Basisdiagnostika (• Tabelle 1). Die diagnostische Arthroskopie, die in der gleichen Sitzung der chirurgischen Rekonstruktion durchgeführt werden sollte, dient der funktionellen Ligamentinspektion und gleichzeitig der Therapie (Glättung, Mikrofrakturing) der häufigen chondralen Begleitläsionen [13, 18]. Die radiologischen Sprunggelenkstressaufnahmen werden nicht mehr zu den Standarddiagnostika gezählt und sollten allmählich aufgegeben werden, da diese keine Zusatzinformationen liefern. Obwohl die MRT die typischen Nachteile einer kostenintensiven sowie einer nicht funktionsanalysierenden Diagnostik aufweist, erlaubt sie den behandelnden Chirurgen dennoch auf nichtinvasive Art eine Beurteilung des medialen Ligamentkomplexes, der Tibialis-posteriorSehne und des Sprunggelenkknorpels [6, 21]. Dies kann bei der Festlegung des geeigneten Behandlungskonzepts und der genauen präoperativen Patientenaufklärung hilfreich sein, muss jedoch wegen falsch-positiver Befunde mit Vorsicht genutzt werden.

\section{Chronische \\ Sprunggelenkinstabilität und fokale OSG-Knorpelläsionen}

Es ist bekannt, dass chronische Sprunggelenkinstabilität generell mit fokalen OSGKnorpelläsionen vereinbar ist. Okuda et al. [17] beschrieben 2005 bei der chronischen lateralen Sprunggelenkinstabilität 63\% chondrale Defekte, Hintermann et al. [13] 2002 bei der chronischen lateralen Sprunggelenkinstabilität $66 \%$ und bei der chronischen medialen Sprunggelenkinstabilität sogar 98\%. Clarke et al. [3] konnten nachweisen, dass bei der Durchtrennung des Lig. deltoideum die Gesamtkontaktfläche im OSG um 15-20\% abnimmt. Deland et al. [4] zeigten, dass eine chronische Deltoidinsuffizienz mit Malalignement zu einer Arthroseentwicklung führen kann. In der Annahme, dass eine chronische ligamentäre Pathomechanik zu pathologisch erhöhten Scherkräften, einer Kontaktflächenreduktion und einem schädlichem intraartikulärem Gelenkdruck führt, sind somit Früherkennung und -behandlung der medialen Sprunggelenkinstabilität und ihrer begleitenden chondralen Läsionen von großer Wichtigkeit, um invalidisierende Langzeitfolgen, wie die Entwicklung eines arthrotischen Sprunggelenks, zu verhindern. 


\section{Rekonstruktionen}

Die alleinige Rekonstruktion der verletzten Bandstrukturen scheint im Normalfall genügend zu sein. Bei einer Rückfußdeformität, namentlich der Valgusfehlstellung der Ferse, dürfte eine korrigierende Kalkaneusosteotomie für einen nachhaltigen Behandlungserfolg erforderlich sein $[10,14]$. Eine laterale Kalkaneusverlängerungsosteotomie ist eher beim abgeflachten Längsgewölbe und symptomatischer Tibialis-posterior-Sehneninsuffizienz vorzuziehen, während bei einer isolierten Valgusstellung des Rückfußes eher eine mediale Kalkaneusverschiebungsosteotomie präferiert werden sollte [10, 11].

\section{Ausblick}

Die in dieser Studie vorgestellte Kasuistik könnte ein übermäßig häufiges Vorkommen der medialen Sprunggelenkinstabilität suggerieren. Dies dürfte allerdings in der täglichen Praxis nicht zutreffen, sondern ist in erster Linie dem Umstand zuzuschreiben, dass der orthopädischen Universitätsklinik Basel eine überaus hohe Anzahl von Patienten mit komplexen und behandlungsresistenten Sprunggelenkinstabilitäten zugewiesen werden. Zudem dürften die in den letzten Jahren gewonnenen Erkenntnisse zu einer genaueren und gezielteren Untersuchung des medialen Sprunggelenks beigetragen haben. Ob die Anzahl an medialen Sprunggelenkinstabilitäten in den letzten Jahren tatsächlich zugenommen hat, wie beispielsweise die Tibialisposterior-Sehnendysfunktion, müsste in spezifischen Studien untersucht werden.

\section{Fazit für die Praxis}

\section{Die vorliegende Studie lässt folgende Schlüsse zu:}

\section{- Die mediale Sprunggelenkinstabili-} tät entsteht wahrscheinlich auf dem Boden eines Pronationstraumas. Typische Beschwerden sind ein Instabilitätsgefühl und ein mediales „giving way" beim Berg- und Treppabgehen. Klinisch findet sich pathognomonisch ein Druckschmerz an der anteromedialen Seite.

- Neben der klinischen Untersuchung ist eine Röntgenaufnahme unter Belastung hilfreich, namentlich um eine Deformität im Seitenvergleich zu erkennen.

- Für die Diagnosesicherung hat sich die Arthroskopie als sehr nützlich erwiesen.

- Die mediale Sprunggelenkinstabilität ist häufig mit einer lateralen Instabilität kombiniert, was zu einer eigentlichen Rotationsinstabilität des Talus in der Malleolengabel führt. Ob und wie zuerst die mediale oder laterale Instabilität bestanden hat, ist weitgehend ungeklärt.

- Die mediale Sprunggelenkinstabilität kann mit einer Tibialis-posterior-Dysfunktion kombiniert vorliegen oder möglicherweise auch auf dem Boden einer Tibialis-posterior-Sehnendysfunktion entstehen.

- Neben Rekonstruktionen der verletzten Bandstrukturen sollten bei etablierten Deformitäten auch ossäre Korrekturen, namentlich Osteotomien am Kalkaneus, erwogen werden.

\section{Korrespondierender Autor}

\section{Dr. V. Valderrabano}

Human Performance Laboratory/Orthopaedics, University of Calgary,

2500 University Drive NW, Calgary, T2N1N4,

Alberta, Kanada

E-Mail:v.valderrabano@kin.ucalgary.ca

\section{Danksagung}

Die Autoren möchten der Schweizerischen Eidgenössischen Sportkommission (ESK, Magglingen, Schweiz) für die finanzielle Unterstützung der Studie im Rahmen des Forschungskonzepts „Sport und Bewegung 2004-2007“ danken.

Interessenkonflikt: Der korrespondierende Autor versichert, dass keine Verbindungen mit einer Firma, deren Produkt in dem Artikel genannt ist, oder einer Firma, die ein Konkurrenzprodukt vertreibt, bestehen.

\section{Literatur}

1. Balen PF, Helms CA (2001) Association of posterior tibial tendon injury with spring ligament injury, sinus tarsi abnormality, and plantar fasciitis on MR imaging. AJR Am J Roentgenol 176:1137-1143

2. Boss AP, Hintermann B (2002) Anatomical study of the medial ankle ligament complex. Foot Ankle Int 23:547-553

3. Clarke HJ, Michelson JD, Cox QG, Jinnah RH (1991) Tibio-talar stability in bimalleolar ankle fractures: a dynamic in vitro contact area study. Foot Ankle 11:222-227
4. Deland JT, de Asla RJ, Segal A (2004) Reconstruction of the chronically failed deltoid ligament: a new technique. Foot Ankle Int 25:795-799

5. Freeman MA (1965) Instability of the foot after injuries to the lateral ligament of the ankle. J Bone Joint Surg [Br] 47:669-667

6. Gazdag AR, Cracchiolo A 3rd (1997) Rupture of the posterior tibial tendon. Evaluation of injury of the spring ligament and clinical assessment of tendon transfer and ligament repair. J Bone Joint Surg [Am] 79:675-681

7. Harper MC (1987) Deltoid ligament: an anatomical evaluation of function. Foot Ankle 8:19-22

8. Hintermann B (1997) Tibialis posterior dysfunction. A review of the problems and personal experience. Foot Ankle Surg 3:61-70

9. Hintermann B, Renggli P (1999) Anatomic reconstruction of the lateral ligaments of the ankle using a plantaris tendon graft in the treatment of chronic ankle joint instability. Orthopade 28:778784

10. Hintermann B, Valderrabano V (2003) Lateral column lengthening by calcaneal osteotomy. Tech Foot Ankle Surg 2:84-90

11. Hintermann B, Valderrabano V, Kundert HP (1999) Lengthening of the lateral column and reconstruction of the medial soft tissue for treatment of acquired flatfoot deformity associated with insufficiency of the posterior tibial tendon. Foot Ankle Int 20:622-629

12. Hintermann B, Valderrabano V, Kundert HP (1999) Lateral column lengthening by calcaneal osteotomy combined with soft tissue reconstruction for treatment of severe posterior tibial tendon dysfunction. Methods and preliminary results. Orthopade $28: 760-769$

13. Hintermann B, Boss A, Schäfer D (2002) Arthroscopic findings in patients with chronic ankle instability. Am J Sports Med 30:402-409

14. Hintermann B, Valderrabano V, Boss A, Trouillier HH, Dick W (2004) Medial ankle instability: an exploratory, prospective study of 52 cases. Am J Sports Med 32:183-190

15. Karlsson J, Bergsten T, Lansinger O, Peterson L (1988) Reconstruction of the lateral ligaments of the ankle for chronic lateral instability. J Bone Joint Surg [Am] 70:581-588

16. Kitaoka HB, Alexander IJ, Adelaar RS, Nunley JA, Myerson MS, Sanders M (1994) Clinical rating systems for the ankle-hindfoot, midfoot, hallux, and lesser toes. Foot Ankle Int 15:349-353

17. Okuda R, Kinoshita M, Morikawa J, Yasuda T, Abe M (2005) Arthroscopic findings in chronic lateral ankle instability: do focal chondral lesions influence the results of ligament reconstruction? Am J Sports Med 33:35-42

18. Schäfer D, Hintermann B (1996) Arthroscopic assessment of the chronic unstable ankle joint. Knee Surg Sports Traumatol Arthrosc 4:48-52

19. Taga I, Shino K, Inoue M, Nakata K, Maeda A (1993) Articular cartilage lesions in ankles with lateral ligament injury. An arthroscopic study. Am J Sports Med 21:120-126

20. Valderrabano V, Hintermann B, Wischer T, Fuhr $P$, Dick W (2001) Michael-Jäger-Preis 2001: Muskuläres Rehabilitationspotential nach später Sehnenrekonstruktion am Beispiel des M. tibialis posterior. Sportorthop Sporttraumatol 17:235-245

21. Valderrabano V, Hintermann B, Wischer T, Fuhr $P$, Dick W (2004) Recovery of the posterior tibial muscle after late reconstruction following tendon rupture. Foot Ankle Int 25:85-95 